\title{
Non-host cells in the pathogenesis of autoimmune disease: a new paradigm?
}

Autoimmune diseases are thought of as disorders in which a body's cells inexplicably attack its own tissues. Usually there is little evidence that anything is abnormal in the body tissues before the immunological attack begins. Drawing together observations from unrelated fields of medicine and science, microchimerism has been proposed as a new consideration in the pathogenesis of some autoimmune disorders. ${ }^{1}$ The term chimerism is used to indicate a body that contains cell populations derived from different individuals and microchimerism refers to low levels of non-host cells. The observations draw from pregnancy immunology, transplantation biology, prenatal diagnostics, and autoimmunity. The key observation that led to the proposal came from investigators working to establish sensitive techniques for prenatal diagnosis. As a result of the application of molecular techniques to prenatal diagnosis it is now known that fetal cells enter the mother's blood early in the course of most pregnancies. In 1996 a report by Dr Diana Bianchi and colleagues cast a potentially new stage for the well recognised female predominance of autoimmunity disease. The investigators found that fetal cells also persist in the maternal circulation for decades after pregnancy completion. ${ }^{2}$ Long term persistence of fetal cells, when considered together with epidemiological observations of autoimmune disease in women and with observations in transplantation biology led me to propose the hypothesis that non-host cells are involved in the pathogenesis of some autoimmune diseases. The hypothesis was proposed in the question "Is some autoimmune disease auto-alloimmune or allo-autoimmune?" The term "allo" refers to non-host cells from an individual of the same species.

In addition to a strong female predominance of autoimmune disease the age specific incidence, for example of scleroderma (SSc) and primary biliary cirrhosis (PBC) in women peaks in middle age-that is after childbearing years. ${ }^{34}$ Interestingly, both of these diseases bear striking similarities to a known condition of human chimerismchronic graft versus host disease (cGvHD) that can occur after haematopoietic stem cell (bone marrow) transplantation. Both SSc and cGvHD exhibit progressive induration of the skin, often involve the gut and lungs and sometimes have accompanying Sjögren's syndrome and myositis. ${ }^{5-7}$ SSc-like manifestations of cGvHD often coexist with cGvHD of the liver and cooccurrence of PBC and SSc has also been described. ${ }^{8}$ Patients with cGvHD disease usually have positive antinuclear antibody tests and antibodies to topoisomerase I and antimitochondrial antibodies have been described. ${ }^{9}{ }^{10}$ Although similarities of cGvHD with autoimmune diseases have been known, and an occasional case of SSc temporally related to pregnancy described, it was the surprising recent finding that fetal microchimerism persists for decades that launched the new model implicating microchimerism in the pathogenesis of some autoimmune disease.

As a first test of the hypothesis that persistent fetal microchimerism is involved in some autoimmune diseases we conducted a prospective study of women with SSc with children born before disease onset and comparable control healthy women. Women were selected who had given birth to at least one son and a quantitative direct polymerase chain reaction (PCR) assay was used to test for a Y-chromosome specific sequence. Sons were studied for technical reasons because of the ability to detect male DNA in a female host. (In contrast with the erroneous statement of an accompanying editorial, nested PCR was not used in any assay). Male DNA was found more frequently and at quantitatively greater levels in women with SSc compared with healthy women. ${ }^{11}$ Among 16 healthy women the range of male cell equivalents was 0 to 2 , mean 0.38 . In contrast, women with SSc had a range of 0 to 61 male cell equivalents, mean 11.1 (table 1 ). The difference between the two groups was statistically significant, $\mathrm{p}=0.0007$. Some women with SSc had levels of male DNA that were higher than that found in most women who are currently pregnant with a normal male fetus, although the patients had given birth to their sons decades previously. The study was blinded for all identifying information including patient versus control and reproductive characteristics. This observation was extended in a study that examined microchimerism in the skin of patients with Ssc. ${ }^{12}$ The study was retrospective, used a nested PCR assay for a Y-chromosome specific sequence, and then evaluated whether patients or controls had previously given birth to a male child. The nested PCR technique is used to detect very small amounts of DNA by conducting two consecutive PCR reactions, the second of which is done on the product from the first (using a second set of primers that are internal to the first set of primers). Eleven women with SSc had positive results compared with none of 68 control women. Nine of the women with positive results had given birth to a male child, one had only daughters but had a prior pregnancy termination, and for one the pregnancy history was unknown. Pregnancy history was unknown for the majority of controls but at least 30\% had sons. Skin biopsy samples were examined using fluorescence in situ hybridisation and male cells reported in SSc patients but not in controls (pregnancy history was not provided for controls studied using in situ hybridisation). Peripheral blood studies, although not quantitative, confirmed the observation that microchimerism is more frequently found in women with SSc than controls.

Persistent cells from a previous pregnancy do not explain autoimmune disease in men or in women who have never

Table 1 Persistent fetal microchimerism in SSc patients and controls assessed by quantitative analysis of male DNA in women with prior birth of at least one son

\begin{tabular}{lll}
\hline & $\begin{array}{l}\text { Women with SSc } \\
(n=17)\end{array}$ & $\begin{array}{l}\text { Healthy women } \\
(n=16)\end{array}$ \\
\hline Male cell equivalent, & $11.1^{\star}$ & 0.38 \\
$\quad$ mean and range & $0-61$ & $0-2$ \\
Age of woman, & 46 & 45 \\
mean and range & $33-64$ & $25-61$ \\
Mean time since most & 15.4 & 18.5 \\
recent son, mean, range & 2 mo-28 y & 2 mo-38 y \\
Other pregnancies & 10 & 9 \\
with daughters & 6 & 3 \\
with fetal loss & &
\end{tabular}

${ }^{\star} \mathrm{p}=0.0007 \mathrm{SSc}$ v Control. 
been pregnant. However, there are alternative sources that could affect these populations. These include engraftment with cells from a twin, from a blood transfusion, or from the mother. The first two of these have previously been described. ${ }^{13}{ }^{14}$ Investigators pursing the goal of using cord blood as a source for marrow transplantation have found that non-shared maternal DNA can be detected in about $40 \%$ of fetal cord blood samples. ${ }^{15}$ In addition, evidence for maternal cells has recently been described in elective terminations from 13 to 25 weeks gestation. ${ }^{16}$ Although microchimerism was not investigated others have described longlasting tolerance to non-inherited maternal HLA antigens. ${ }^{18}$ To pursue the possibility that maternal cells might sometimes persist long term we investigated a 47 year old SSc patient. HLA maternal specific PCR tests were conducted on DNA extracted from blood samples from the patient. Maternal specific DNA was detected in samples from the patient from three separate draw dates. Sequencing confirmed the product to be identical to the maternal HLA allele. ${ }^{19}$ Thus maternal cells may be added to other potential sources of persistent microchimerism.

HLA molecules allow an individual to distinguish "self" from "other", are critical determinants of immune responses, and specific HLA class II molecules have been described in association with a variety of autoimmune diseases. In a corollary hypothesis ${ }^{1}$ it was proposed that prior birth of an HLA compatible child might increase risk of subsequent autoimmune disease in the mother. For example, if a child's cell persisted that was HLA similar but not identical, could "autoimmune" disease result if later in life a trigger such as a virus or environmental agent activated the cell? To answer this question HLA studies were conducted for 136 individuals from 21 SSc families and 130 individuals from 32 control families, with the inclusion requirement that all children be willing to participate. ${ }^{11}$ The study design criteria is essential because the hypothesis is that prior birth of at least one HLA compatible child is the risk factor, not multiparity or number of children, so that non-inclusion of a child could result in the misclassification of a patient or a control. Women with SSc were almost nine times more likely to have given birth to a child prior to disease onset that was compatible for the basic DR families $\mathrm{DRB} 1^{\star} 01$ through $\mathrm{DRB} 1{ }^{\star} 14 \mathrm{DRB} 1$, and 19 times more likely if the child was compatible because of DRB1 homozygosity (table 2). No association was observed for HLA class I antigens. The likelihood of cGvHD in patients who undergo haematopoietic stem cell transplantation is greater with increasing HLA incompatibility from the perspective of the donor and interestingly, fatal GvHD has been described in immune competent recipients of blood transfusions when the donor is a family member who is HLA compatible because of homozygosity. ${ }^{20}$

By analogy with manifestations of cGvHD other diseases that might be investigated include PBC, Sjögren's syndrome, myositis, and systemic lupus. In early reports investigating microchimerism in other diseases Reed et al have described microchimerism in children with

Table 2 HLA compatibility of a previously born child for the class II loci $D R B 1, D Q A 1$ and $D Q B 1$ and risk of subsequent SSc in the mother

\begin{tabular}{|c|c|c|c|c|}
\hline \multirow[b]{2}{*}{ Locus } & Scleroderma $(n=21)$ & Controls $(n=32)$ & \multirow[b]{2}{*}{ Odds ratio } & \multirow[b]{2}{*}{ p Value } \\
\hline & $\begin{array}{l}\text { HLA compatible } \\
\text { Number (\%) }\end{array}$ & $\begin{array}{l}\text { HLA compatible } \\
\text { Number (\%) }\end{array}$ & & \\
\hline $\mathrm{DRB}_{1}$ & $13(62 \%)$ & $5(16 \%)$ & 8.78 & 0.001 \\
\hline $\mathrm{DRB} 1_{2}$ & $9(43 \%)$ & $3(9 \%)$ & 7.25 & 0.012 \\
\hline DQA1 & $16(76 \%)$ & $12(38 \%)$ & 4.17 & 0.031 \\
\hline DQB1 & $13(62 \%)$ & $11(34 \%)$ & 3.10 & 0.091 \\
\hline
\end{tabular}

${ }^{\star}$ For $\mathrm{DRB} 1_{1}$, attributable to homozygosity $\mathrm{OR}=19.08, \mathrm{p}=0.003 . \mathrm{DRB} 1_{1}$ defines $\mathrm{DR} 1$ through $\mathrm{DR} 14$ and $\mathrm{DRB}_{2}$ the specific allele within these families. Adapted from Nelson et al. ${ }^{11}$ dermatomyositis. ${ }^{21}$ Maternal microchimerism may be implicated in some cases of systemic lupus based on an experimental model of this disease that involves the induction of chimerism by introduction of parental cells into the progeny. ${ }^{22}{ }^{23}$ Microchimerism could also play a part in diseases that occur during pregnancy and pregnancy induced modulation of disease activity in women who have a pre-existing autoimmune disease. In earlier studies we found that remission of rheumatoid arthritis during pregnancy was associated with fetal-maternal HLA disparity for HLA class II antigens, particularly for HLA-DQA $1 .{ }^{24}$ In a study by Aractingi et al microchimerism was investigated in women suffering from puritic eruption of pregnancy, a skin disorder that usually occurs during pregnancy. In an elegant and carefully done study the authors demonstrated microchimerism in skin biopsy samples from patients but not from matched control women. ${ }^{25}$

Mechanism(s) by which microchimerism might contribute to the pathogenesis of autoimmune diseases such as SSc have not been investigated. Studies have investigated chimerism in organ transplant recipients and patients with cGvHD. Starzl and colleagues proposed the interesting hypothesis that donor cell microchimerism facilitates graft acceptance as an explanation for chimerism in recipients of organ transplants who can withdraw immunosuppressive medications without rejection. ${ }^{26}$ This hypothesis is appealing as an explanation for microchimerism during pregnancy as pregnancy can be viewed as tolerance of a fetal allograft. However, an explanation for why fetal microchimerism should persist for many years after pregnancy or cause disease is not evident. Mechanisms that contribute to cGvHD have been summarised. ${ }^{27}$ These include donor $\mathrm{T}$ cells that are not deleted and are not tolerised to the host, damage to the thymus by acute GvHD so that maturing $T$ cells become self reactive and a shift to Th2 cytokines. In SSc patients it was suggested that CD3+ fetal cells direct a GvHD reaction on maternal tissues, however, the basis for the suggestion was detection of male cells among CD3+ selected cells in a few SSc patients without an appropriate control group. ${ }^{12}$ Moreover, the original study of healthy normal controls by Bianchi et al included description of microchimerism in CD3 and studies since then have confirmed that microchimerism is common in CD3 in healthy normal controls. ${ }^{28}$ At least two other reasons indicate direct analogies with $c G v H D$ require caution. These are clinical differences and quantitative differences of chimerism between the SSc and cGvHD. Although there are numerous similarities in the two disorders there are also differences, in clinical features such as Raynaud's phenomenon and renal disease and also in histopathology. ${ }^{29}{ }^{30} \mathrm{An}$ even more important difference between SSc and cGvHD is the quantitative difference of chimerism between the disorders. The non-host to host cell ratio can be estimated from our quantitative study at most as less than one in a million white blood cells, or if lymphocytes, one in 500 000; in contrast in cGvHD donor cells replace circulating host cells. Large numbers of chimeric cells could be sequestered in disease affected tissues, however, studies of skin specimens ${ }^{12}$ suggest that levels of microchimerism are also not marked in skin.

Small numbers of non-host cells could have a disproportionate effect if the role was that of dysregulating host cells, allowing damage by autoreactive host cells, and/or a shift in cytokine milieu with subsequent damage. Interference with host immunoregulatory pathways could be mediated by non-host cells or by non-host peptides. Presentation of non-host peptides by host cells to other host cells (the "indirect" pathway of allorecognition) is thought to play a part in chronic allograft rejection that has some similarities with SSc. ${ }^{31}$ Alternatively, non-host cells and host cells 
could interact directly, for example, if a small number of CD8+ fetal cells coopted help from CD4+ host cells ${ }^{32}$ a process that could be facilitated by HLA compatibility. Non-responsiveness of maternal cells could be induced by fetal "veto" cells, an antigen presenting cell that inactivates a $\mathrm{T}$ cell with which it interacts. ${ }^{33}$ These possibilities are not mutually exclusive and more than one mechanism could be involved.

In summary, recent studies indicate there is bidirectional traffic of cells during normal human pregnancy. Fetal cells have been found to persist in the maternal peripheral blood for many years after pregnancy. Many autoimmune diseases are more prevalent in women, some of which also have a peak incidence following childbearing years. A known condition of chimerism, cGvHD has clinical similarities to some autoimmune diseases, notably SSc, PBC, Sjögren's syndrome, and sometimes myositis and systemic lupus. Results of early studies offer support to the hypothesis that that microchimerism may be involved in the pathogenesis of some autoimmune disease. However, microchimerism is common in healthy normal people emphasising the importance of careful study design and selection of appropriate control populations. If microchimerism does contribute to the pathogenesis of some autoimmune disorders it is likely that new therapeutic strategies could be developed.

J LEE NELSON

Program in Human Immunogenetics, Fred Hutchinson Cancer Research Center, and Rheumatology, University of Washington, Seattle, WA, USA

Correspondence to: Dr J L Nelson, Immunogenetics D2-100, Fred

Hutchinson Cancer Research Center, 1100 Fairview Ave North, Seattle, WA 98109-1024, USA.

Funding: support for some of the studies described was provided by National Institutes for Health research grants AI41721, AI38583 and HD35927.

1 Nelson JL. Maternal-fetal immunology and autoimmune disease. Is some autoimmune disease auto-alloimmune or allo-autoimmune? Arthritis Rheum 1996;39:191-4.

2 Bianchi DW, Zickwolf GK, Weil GJ, Sylvester S, DeMaria MA. Male fetal progenitor cells persist in maternal blood for as long as 27 years postpartum. Proc Natl Acad Sci 1996;93:705-8.

3 Silman AJ. Epidemiology of scleroderma. Ann Rheum Dis 1991;50:4:887-93.

4 Danielsson A, Boqvist L, Uddenfeldt P. Epidemiology of primary biliary cirrhosis in a defined rural population in the northern part of Sweden. Heprhosis in a defined rural
tology $1990 ; 11: 458-64$.

5 Lawley T, Peck G, Moutsopoulos H, Gratwohl A, Deisseroth A. Scleroderma, Sjögren-like syndrome, and chronic graft-versus-host disease. Ann Intern Med 1977;87:707-9.

6 Gratwhol A, Moutsopoulos H, Chused T, Akizuki M, Wolf R, Sweet J, et al. Sjögren-type syndrome after allogeneic bone marrow transplantation. Ann Intern Med 1977, 87:703-6.

7 Urbano-Marquez A, Estruch R, Grau J, Granena A, Martin-Ortega E, Palou $\mathrm{J}$, et al. Inflammatory myopathy associated with chronic graft-versus-host disease. Neurology 1986;36:1091-3.

8 Culp KS, Fleming CR, Duffy J, Baldus WP, Dickson ER. Autoimmune associations in primary biliary cirrhosis. Mayo Clin Proc 1982;57:365-70.
9 Bell SA, Faust H, Mittermuller J, Kolb HJ, Meurer M. Specificity of antinuclear antibodies in scleroderma-like chronic graft-versus-host disease: clini$\mathrm{cal}$ correlation and histocompatibility locus antigen association. $\mathrm{Br} \mathrm{J}$ Dermatol 1996;134:848-54.

10 Siegert W, Stemerowicz R, Hopf U. Antimitochondrial antibodies in patients with chronic graft-versus-host disease. Bone Marrow Transplant 1992;10: 221-7.

11 Nelson JL, Furst DE, Maloney S, Gooley T, Evans PC, Smith A, et al. Microchimerism and HLA-compatible relationships of pregnancy in scleroderma. Lancet 1998;351:559-62.

12 Artlett CM, Smith JB, Jimenez, SA. Identification of fetal DNA and cells in skin lesions from women with systemic sclerosis. N Engl J Med 1998;338: skin lesions

13 De Moor G, De Bock G, Noens L, De Bic S. A new case of human chimerism detected after pregnancy: 46,XY karyotype in the lymphocytes of a woman. Acta Clin Belg 1988;43:231-5.

14 Lee TH, Paglieroni T, Ohto H, Holland PV, Busch MP. Survival of donor leukocyte subpopulations in immunocompetent transfusion recipients: Frequent long-term microchimerism in severe trauma patients. Blood 1999;9:3127-39.

15 Lo YMD, Lo ESF, Watson N, Noakes L, Sargent IL, Thilaganathan B, et al. Two-way cell traffic between mother and fetus: biologic and clinical implications. Blood 1996;88:4390-5.

16 Petit T, Dommergues M, Socie G, Dumez Y, Gluckman E, Brison O. Detection of maternal cells in human fetal blood during the third trimester of pregnancy using allele-specific PCR amplification. Br J Haematol 1997; 100:767-71.

17 Lo ESF, Lo YMD, Hjelm NM, Thilaganathan B. Transfer of nucleated maternal cells into fetal circulation during the second trimester of pregnancy. [Letter]. Br J Haematol 1998;100:605-6.

18 Claas FHJ, Gijbels Y, van der Velden-de Munck J, van Rood JJ. Induction of $\mathrm{B}$ cell unresponsiveness to noninherited maternal HLA antigens during B cell unresponsiveness to noninherited maternal HLA antigens during

19 Maloney S Evans P, Storek J, Smith AJ, Furst DE, Nelson JL. Evidence for persistent microchimerism with maternal cells in a patient with scleroderma. [Abstract]. Arthritis Rheum 1997;40:S1010.

20 McMilin KD, Johnson RL. HLA homozygosity and the risk of related-donor transfusion-associated graft-versus-host disease. Transfus Med Rev 1993, 7:37-41.

21 Reed AM, Shock LP, Picornell YJ. Microchimerism in children with juvenile dermatomyositis. [Abstract]. Arthritis Rheum 1998;41:S264.

22 Nelson JL. Microchimerism and autoimmune disease. N Engl J Med 1998; 338:1224-5.

23 Gleichman E, Van Elven H, Van der Veen JP. A systemic lupus erythematosus (SLE)-like disease in mice induced by abnormal T-B cell cooperation. Eur J Immunol 1982;12:152-5.

24 Nelson JL, Hughes KA, Smith AG, Nisperos BB, Branchaud AM, Hansen JA. Maternal-fetal disparity in HLA class II alloantigens and the pregnancy-induced amelioration of rheumatoid arthritis. N Engl J Med pregnancy-induced
1993;329:466-71.

25 Aractingi S, Berkane N, Bertheau P, Le Goue C, Dausset J, Uzan S, et al. Fetal DNA in skin of polymorphic eruptions of pregnancy. Lancet 1999;352:1898-901

26 Starzl TE, Demetris AJ, Murase N, Trucco M, Thomson AW, Rao AS. The lost chord: microchimerism and allograft survival. Immunol Today 1996; 17:577-84.

27 Hakim FT, Mackall CL. The immune system: effector and target of graft-versus-host disease. In: Ferrara JL, Deeg HJ, Burakof ST, eds. Graftvs-host disease. 2nd ed. New York: Marcel Deker, 1997:274-89.

28 Evans PC, Lambert N, Maloney S, Furst DE, Moore JM, Nelson JL. Longterm fetal microchimerism in peripheral blood mononuclear cell subsets in healthy women and women with scleroderma. Blood 1999;93:1-6.

29 Aractingi S, Dausset J, Carosella ED. Chimerism in scleroderma. [Letter] Lancet 1998;351:1996.

30 Nelson JL. Chimerism and scleroderma, reply. [Letter]. Lancet 1998;351: 1887.

31 Pandey JP, LeRoy EC. Human cytomegalovirus and the vasculopathies of autoimmune diseases (especially scleroderma) allograft rejection, and coronary resstenosis. Arthritis Rheum 1998;41:10-15.

32 Suzuki K, Narita T, Yui R, Asakura H, Fujiwara M. Mechanism of the induction of autoimmune disease by graft-versus-host reaction. Role of CD8+ cells in the development of hepatic and ductal lesions induced by CD4+ cells in MHC class I plus II different host. Lab Invest 1994;70: 609-19.

33 Miller RG. The veto phenomenon and T-cell regulation. Immunol Today 1986;7:112-14. 